\title{
A generalization of the $S$-function method applied to a Duffing-Van der Pol forced oscillator
}

\author{
L.G.S. Duarte ${ }^{\mathrm{a}}$, L.A.C.P. da Mota ${ }^{\mathrm{a}, *}$ \\ ${ }^{a}$ Universidade do Estado do Rio de Janeiro, Instituto de Física, Depto. de Física \\ Teórica, 20559-900 Rio de Janeiro - RJ, Brazil
}

\begin{abstract}
In [1, 2] we have developed a method (we call it the $S$-function method) that is successful in treating certain classes of rational second order ordinary differential equations (rational 2ODEs) that are particularly 'resistant' to canonical Lie methods and to Darbouxian approaches. In this present paper, we generalize the $S$-function method making it capable of dealing with a class of elementary 2ODEs presenting elementary functions. Then, we apply this method to a Duffing-Van der Pol forced oscillator, obtaining an entire class of first integrals.
\end{abstract}

Keywords: Elementary 2ODEs, $S$-function method, Duffing-Van der Pol forced oscillator

\section{Introduction}

The most general methods for finding closed-form solutions and global first integrals of dynamical systems derive from Lie [3] and Darboux [4] approaches. Thus, it is unnecessary to stress the importance of procedures that compute Lie symmetries (symmetry continuous groups) or Darboux polynomials (that define algebraic invariant curves or surfaces) associated with a given differential equation (DE) or a system of DEs. However, even these powerful approaches sometimes fail (this usually happens in cases where the

*Corresponding author

L.G.S. Duarte and L.A.C.P. da Mota wish to thank Fundação de Amparo à Pesquisa do Estado do Rio de Janeiro (FAPERJ) for a Research Grant.

Email addresses: lgsduarte@gmail.com.br (L.G.S. Duarte), lacpdamota@uerj.br or lacpdamota@dft.if.uerj.br (L.A.C.P. da Mota) 
Darboux polynomials have a relatively high degree, say $\geq 4$, and / or the Lie symmetries are dynamical or nonlocal). Therefore, there is a constant search for generalizations of these methods, which, in turn, leads to the definition of a series of concepts and alternative procedures to attack these more complicated cases. Among these, we highlight: [5, 6, 7, 8, 9, 10, 11, 12, 13, 14] in the 'symmetry side' and [15, 16, 17, 18, 19, 20, 21, 22, 23, 24] in the 'Darbouxian side'.

In 1, 2] we have developed an algorithm to find Liouvillian first integrals of rational second order ordinary differential equations (rational 2ODEs). This procedure is based on the computation of the $S$-function 1 that is closely linked to the $\lambda$-symmetry concept created by Muriel and Romero in [7]. In this paper we generalize the method developed in [1, 2] and use it to find first integrals of a forced Duffing-Van der Pol oscillator [27, 26]. As the determination of the $S$-function is based on a Darbouxian type technique, we can do, during the search process, an integrability analysis in the same way we did in [19].

In the first section we will briefly review the algorithm described in [2]. Next, we present a generalization of the method that allows us to use the algorithm to deal with 2ODEs presenting elementary functions. In section 3 we use the method to make an analysis of the integrability region of the parameters present in a forced Duffing-Van der Pol oscillator. Finally we present our conclusions and ways to extend our work.

\section{The $S$-function method}

In this section, we will introduce some basic concepts involving rational 2ODEs and some results (for the proofs see [2]) that allowed for the construction of an algorithm to search for Liouvillian first integrals.

\subsection{The basic concepts and results}

Consider the rational 2ODE given by

$$
z^{\prime}=\frac{d z}{d x}=\phi(x, y, z)=\frac{M(x, y, z)}{N(x, y, z)},\left(z \equiv y^{\prime}\right),
$$

where $M$ and $N$ are coprime polynomials in $(x, y, z)$.

\footnotetext{
${ }^{1}$ The $S$-function was created in [15] where we generalize the Prelle and Singer method 25] to deal with rational 2ODEs.
} 
Definition 2.1. A function $I(x, y, z)$ is called first integral of the $2 O D E$ (1) if $I$ is constant over the solutions of (1).

Definition 2.2. Let I be a first integral of the 2ODE (11). The function defined by $S:=I_{y} / I_{z}$ is called a $\boldsymbol{S}$-function associated with the 2ODE through the first integral $I$.

Theorem 2.1. Let $S$ be a $S$-function associated with the $2 \mathrm{ODE} z^{\prime}=\phi(x, y, z)$. Then $S$ obeys the following equation:

$$
D_{x}[S]=S^{2}+\phi_{z} S-\phi_{y} .
$$

Definition 2.3. Let I be a first integral of the 2ODE (11) and let $S(x, y, z)$ be the S-function associated with (11) through $I$. The first order ordinary differential equation defined by

$$
\frac{d z}{d y}=-S(x, y, z)
$$

where $x$ is taken as a parameter, is called 1ODE associated with the 2ODE (11) through $I$.

Theorem 2.2. Let $I$ be a first integral of the 2ODE (11) and let $S(x, y, z)$ be the S-function associated with (11) through $I$. Then $I(x, y, z)=C$ is a general solution of the 1ODE associated with (1) through $I$.

Remark 2.1. Note that Theorem 2.2 does not imply that, if we solve the 1ODE associated (3), we would obtain $I(x, y, z)=C$. The reason is that the variable $x$ (the independent variable of the $2 \mathrm{ODE}(1))$ is just a parameter in the 1ODE (3).

Remark 2.2. Since any function of $x$ is an invariant for the operator $\partial_{y}-$ $S \partial_{z}$, i.e., $\left(\partial_{y}-S \partial_{z}\right)[F(x)]=0$, the relation between a general solution $H(x, y, z)=K$ of the 1ODE (3) and the first integral $I(x, y, z)$ of the 2ODE (11) is given by $I(x, y, z)=\mathcal{F}(x, H)$, such that the function $\mathcal{F}$ satisfies

$$
D_{x}[I]=\frac{\partial \mathcal{F}}{\partial x}+\left(\frac{\partial H}{\partial x}+z \frac{\partial H}{\partial y}+\phi \frac{\partial H}{\partial z}\right) \frac{\partial \mathcal{F}}{\partial H}=0 .
$$




\subsection{Construction of a method}

If the 2ODE (11) presents a Liouvillian first integral $I$ such that the as-

sociated $S$-function is given by $S=\frac{P}{N}$ then, substituting it into the 1PDE $D_{x}[S]=S^{2}+\phi_{z} S-\phi_{y}$ we obtain:

$$
-P^{2}-\left(N_{x}+z N_{y}+M_{z}\right) P+D[P]-M N_{y}+M_{y} N=0,
$$

where $D \equiv N D_{x}=N \partial_{x}+z N \partial_{y}+M \partial_{z}$. Then we construct a polynomial $\mathcal{P}$ with undetermined coefficients and substitute it in (5). After, we collect the resulting polynomial equation in the variables $x, y, z$ and equate the coefficients of each monomial to zero, obtaining a system of equations. If this system presents a solution we will have found $S$ and we can construct the associated 1ODE (and try to solve it). From the solution of the associated 1ODE we can find the first integral $I(x, y, z)$ by solving the characteristic system of de 1PDE (4). So, we can propose the following algorithm:

\section{Algorithm 2.1.}

1. Let $n_{\max }=\max (\operatorname{degree}(M)-1$, degree $(N))$.

2. Let $n=0$.

3. Let $n=n+1$.

4. if $n>n_{\max }$ then FAIL.

5. Construct the $D_{x}$ operator.

6. Construct a generic polynomial $\mathcal{P}$ of degree $n$ in $(x, y, z)$ with undetermined coefficients $a_{i}$.

7. Substitute $\mathcal{P}$ in equation (5), collect the resulting polynomial equation in the variables $x, y, z$ and equate the coefficients of each monomial to zero, obtaining a system $\mathcal{A}$ of algebraic equations.

8. Solve the system $\mathcal{A}$ with respect to $\left\{a_{i}\right\}$. If no solution is found, then go to step 3.

9. Substitute the solution in $\mathcal{P}$ (obtaining $P$ ) and construct $S=P / N$.

10. Construct the associated $1 O D E\left(\frac{d z}{d y}=-\frac{P}{N}\right)$ and try to solve it to obtain a solution $H(x, y, z)=C$. If no solution is found, then go to step 3.

11. Solve $h=H(x, y, z)$ for one of the variables $x, y$ or $z$ (or any operand belonging to $H$ and to $\left.D_{x}[H]\right)$ and substitute it in the 1PDE (4). Then, try to solve the characteristic equation - a 1ODE in $(x, h)$ - to obtain $\mathcal{F}(x, h)=K$. If no solution is found, then go to step 3 .

12. Construct the first integral $I=\mathcal{F}(x, H)$. 


\section{A generalization of the $S$-function method to deal with 2ODEs presenting elementary functions}

The main idea is to consider 2ODEs of the form

$$
z^{\prime}=\frac{d z}{d x}=\phi(f(x), y, z)=\frac{M(g(x), y, z)}{N(g(x), y, z)},\left(z \equiv y^{\prime}\right),
$$

where $M$ and $N$ are coprime polynomials in $(g(x), y, z)$. If we perform a change of variables such that $u=f(x)$ into the 2ODE (6) we have

$$
\begin{aligned}
\frac{d y}{d x} & =\frac{d y}{d u} \frac{d u}{d x}=\frac{d y}{d u} f^{\prime}(x), \\
\frac{d^{2} y}{d x^{2}} & =\frac{d}{d x}\left(\frac{d y}{d x}\right)=\frac{d}{d x}\left(\frac{d y}{d u} f^{\prime}(x)\right)= \\
& =f^{\prime \prime}(x) \frac{d y}{d u}+f^{\prime}(x)^{2} \frac{d^{2} y}{d u^{2}} .
\end{aligned}
$$

Substituting in the 2ODE (6) we obtain

$$
f^{\prime \prime}(x) \frac{d y}{d u}+f^{\prime}(x)^{2} \frac{d^{2} y}{d u^{2}}=\frac{M\left(g(x), y, \frac{d y}{d u} f^{\prime}(x)\right)}{N\left(g(x), y, \frac{d y}{d u} f^{\prime}(x)\right)} .
$$

The idea is that if the function $f(x)$ is such that $g(x)=h_{0}(u)$ and $f^{\prime}(x)=$ $h_{1}(u)$, where $h_{0}$ and $h_{1}$ are rational functions of $u$, it is clear that the 2ODE (9) will be rational (for in that case, $f^{\prime \prime}(x)$ will also be a rational function of $u$ ). In this way, we can apply the $S$-funtion method to it. We can see that if $g(x)$ is an elementary function, this will work in many cases. For example, let $g(x)=\left(1+\mathrm{e}^{2 x}\right)$ : in this case choosing $f(x)=\mathrm{e}^{x}$ we have $g(x)=1+u^{2}$, $f^{\prime}(x)=u, f^{\prime \prime}(x)=u$.

\section{A first integral for a forced Duffing Van der Pol oscillator}

The Duffing-Van der Pol's differential equation [27, 26] is an important mathematical model for representing dynamical systems having a single unstable fixed point and a single stable limit cycle. This kind of equation represents many physical phenomena and, like many others nonlinear problems, the forced Duffing-Van der Pol oscillator does not have global analytical first 
integrals for all values of the parameters [28, 29]. Therefore, it is very important to have tools that can analyse the integrability range of parameters' values. This has already been done for the force free Duffing Van der Pol oscillator [30, 31]. With the generalization just developed, we can apply the $S$-function method to achieve this purpose. Consider the following forced Duffing-Van der Pol oscillator DE:

$$
\frac{d^{2} x}{d t^{2}}-\mu\left(1-x^{2}\right) \frac{d x}{d t}+\alpha x+\beta x^{3}=f \cos (\omega t),
$$

where $x$ represents the displacement from the equilibrium, $\mu>0$ is the damping parameter and $f \cos (\omega t)$ is the periodic driving function of time.

For this 2ODE, we can see that $g(t)=\cos (\omega t)$. So, if we choose $\tau=$ $f(t)=\mathrm{e}^{i \omega t}$, we will have $f^{\prime}(t)=i \omega \mathrm{e}^{i \omega t}=i \omega \tau, f^{\prime \prime}(t)=-\omega^{2} \mathrm{e}^{i \omega t}=-\omega^{2} \tau$ and $g(t)=\cos (\omega t)=(\tau+1 / \tau) / 2$. The transformation given by the eqs. (7,8) is

$$
\begin{aligned}
\frac{d x}{d t} & =\frac{d x}{d \tau} \frac{d \tau}{d t}=\frac{d x}{d \tau} f^{\prime}(t)=\frac{d x}{d \tau} i \omega \tau, \\
\frac{d^{2} x}{d t^{2}} & =\frac{d}{d t}\left(\frac{d x}{d t}\right)=\frac{d}{d t}\left(\frac{d x}{d \tau} f^{\prime}(t)\right)= \\
& =f^{\prime \prime}(t) \frac{d x}{d \tau}+f^{\prime}(t)^{2} \frac{d^{2} x}{d \tau^{2}}= \\
& =-\omega^{2} \tau \frac{d x}{d \tau}-\omega^{2} \tau^{2} \frac{d^{2} x}{d \tau^{2}}
\end{aligned}
$$

Substituting this in the 2ODE (10) we obtain:

$$
\frac{d^{2} x}{d \tau^{2}}=\frac{\left(m \tau^{2}\left(x^{2}-1\right)-\omega^{2} \tau^{2}\right) \frac{d x}{d \tau}+b x^{3} \tau+a x \tau+f\left(\tau^{2}+1\right)}{\omega^{2} \tau^{3}} .
$$

Applying the Algorithm 2.1 to the 2ODE (13) we obtain the following $S$ function

$$
S=\frac{-m x^{2}+k}{\omega^{2} \tau}
$$

where the parameter $k$ was introduced (by the method) in the search for a rational $S$-function associated with the 2ODE (13). The present solution was 
found accompanied by the following relation between the 2ODE parameters:

$$
\left\{a=\frac{k(k-m)}{\omega^{2}}, b=-\frac{1}{3} \frac{m(k-m)}{\omega^{2}}, f=f, m=m, \omega=\omega\right\} .
$$

Applying the relation (15) to the 2ODE (13) we obtain

$$
\frac{d^{2} x}{d \tau^{2}}=\frac{\left(m \tau^{2}\left(x^{2}-1\right)-\omega^{2} \tau^{2}\right) \frac{d x}{d \tau}-\frac{m(k-m)}{3 \omega^{2}} x^{3} \tau+\frac{k(k-m)}{\omega^{2}} x \tau+f\left(\tau^{2}+1\right)}{\omega^{2} \tau^{3}} .
$$

Continuing the application of Algorithm 2.1 to the 2ODE (16) we will obtain:

- The 1ODE associated is given by

$$
\frac{d v}{d x}=\frac{-m x^{2}+k}{\omega^{2} \tau},\left(v \equiv \frac{d x}{d \tau}\right)
$$

- The solution of the 1ODE associated is

$$
H(x, v)=\frac{-m x^{3}+3 \omega^{2} \tau v+3 k x}{3 \omega^{2} \tau}=C .
$$

- The first integral is

$$
I(\tau, x, v)=\left(v \tau-\frac{m x^{3}}{3 \omega^{2}}+\frac{k x}{\omega^{2}}+\frac{f \tau}{-\omega^{2}+k-m}+\frac{f}{\tau\left(\omega^{2}+k-m\right)}\right) \tau^{\frac{m-k}{\omega^{2}}} .
$$

Turning back to the original variables, we finally have

$I(t, x, \dot{x})=\left(\frac{k x}{\omega^{2}}-i \frac{3 \dot{x}+\mu x^{3}}{3 \omega}+\frac{f \mathrm{e}^{i \omega t}}{k-\omega^{2}-i \omega \mu}+\frac{f \mathrm{e}^{-i \omega t}}{k+\omega^{2}-i \omega \mu}\right) \mathrm{e}^{-\left(\mu+i \frac{k+\omega^{2}}{\omega}\right) t}$.

\section{Conclusion}

Since the introduction of the $S$-function [15], we have been finding new applications and developments for it (other people as well, the first ones were [17]). Recently, we have developed a procedure to algorithmically search for first order Liouvillian differential invariants for 2ODEs [1, 2]. 
Here, we introduce a new method that uses the $S$-function in an algorithm to deal with (a class of) 2ODEs presenting functions. The method introduced in [1, 2] deal with rational 2ODEs. We illustrate the importance of this development by tackling the forced Duffing-Van der Pol oscillator.

The method allows for an integrability analysis and we have managed to find a set of values for the parameters and find a class of first order invariants for this well known problem.

We hope to have shown to the reader that our method, hereby presented, has the potential to find new solutions to very interesting problems, such as the one we have presented.

\section{References}

\section{Referências}

[1] L.G.S.Duarte, L.A.C.P. da Mota and E.Nunez, Finding first order differential invariants through the S-function, Computer Physics Communications, Vol. 207, 542-544, (2016).

[2] J. Avellar, M.S. Cardoso, L.G.S. Duarte, L.A.C.P. da Mota Dealing with Rational Second Order Ordinary Differential Equations where both Darboux and Lie Find It Difficult: The S-function Method, http://arxiv.org/abs/1707.09007.

[3] S. Lie, Theorie der Transformationsgruppen, Vol. I, II, III, Chelsea, New York, (1970).

[4] G. Darboux, Mémoire sur les équations différentielles algébriques du premier ordre et du premier degré (Mélanges), Bull. Sci. Math. 2ème série 2, 60-96, 2, 123-144, 2, 151-200 (1878).

[5] B. Abraham-Shrauner, K.S. Govinder and P.G.L Leach, Integration of second order ordinary differential equations not possessing Lie point symmetries. Phys. Lett. A, 203, 169-74, (1995).

[6] K.S. Govinder and P.G.L Leach, A group theoretic approach to a class of second-order ordinary differential equations not possesing Lie point symmetries. J. Phys. A: Math. Gen., 30, 2055-68, (1997).

[7] C. Muriel and J.L. Romero, New methods of reduction for ordinary differential equations, IMA J. Appl. Math., 66(2), 111-125, (2001). 
[8] C. Muriel and J.L. Romero, $C^{\infty}$-Symmetries and reduction of equations without Lie point symmetries, J. Lie Theory, 13(1), 167-188, (2003).

[9] M.C. Nucci, Lie symmetries of a Painlevé-type equation without Lie symmetries, Journal of Nonlinear Mathematical Physics, 15(2), 205211, (2008).

[10] C. Muriel and J.L. Romero, Nonlocal Symmetries, Telescopic Vector Fields and $\lambda$-Symmetries of Ordinary Differential Equations, SIGMA, 8, 106, (2012).

[11] M.S. Bruzón, M.L. Gandarias and M. Senthilvelan, Nonlocal symmetries of Riccati and Abel chains and their similarity reductions. Journal of Mathematical Physics, 53, 023512 (2012).

[12] G. Bluman and R. Dridi, New solutions for ordinary differential equations, Journal of Symbolic Computation, 47, 1, (2012).

[13] C. Muriel and J.L. Romero, The $\lambda$-symmetry reduction method and Jacobi last multipliers, Commun Nonlinear Sci Numer Simulat, 19 807-820, (2014).

[14] C. Muriel, J. L. Romero and A. Ruiz, $\lambda$-Symmetries and integrability by quadratures, IMA Journal of Applied Mathematics, 82, 5, (2017).

[15] L.G.S. Duarte, S.E.S.Duarte, L.A.C.P. da Mota and J.F.E. Skea, Solving second order ordinary differential equations by extending the PrelleSinger method, J. Phys. A: Math.Gen., 34, 3015-3024, (2001).

[16] L.G.S. Duarte, S.E.S.Duarte and L.A.C.P. da Mota, Analyzing the Structure of the Integrating Factors for First Order Ordinary Differential Equations with Liouvillian Functions in the Solution, J. Phys. A: Math. Gen., 35, 1001-1006, (2002).

[17] V.K. Chandrasekar, M. Senthilvelan and M. Lakshmanan, On the complete integrability and linearization of certain second order nonlinear ordinary differential equations. Proceedings of the Royal Society London, Series A, 461, 2060, (2005).

[18] J. Avellar, L.G.S. Duarte, S.E.S.Duarte and L.A.C.P. da Mota, Determining Liouvillian first integrals for dynamical systems in the plane, Computer Physics Communications, 177, 584-596, (2007). 
[19] L.G.S.Duarte and L.A.C.P.da Mota, Finding Elementary First Integrals for Rational Second Order Ordinary Differential Equations, J. Math. Phys., 50, (2009).

[20] A. Ferragut and H. Giacomini, A new algorithm for finding rational first integrals of polynomial vector fields Qual. Theory Dyn. Syst., 9, (2010).

[21] C. Christopher, J. Llibre, C. Pantazi and S. Walcher, Inverse Problems in Darboux' Theory of Integrability, Acta Applicandae Mathematicae, 120, 1, (2012).

[22] P.B. Acosta-Humánez, J.T. Lázaro, J.J. Morales-Ruiz and C. Pantazi, On the integrability of polynomial vector fields in the plane by means of Picard-Vessiot theory., Discrete Contin. Dyn. Syst., 35, 5, (2015).

[23] X. Zhang, Liouvillian integrability of polynomial differential systems, Trans. Amer. Math. Soc., 368, 1, (2016).

[24] A. Bostan, G. Chèze, T. Cluzeau and J.-A. Weil, Efficient algorithms for computing rational first integrals and Darboux polynomials of planar polynomial vector fields, Math. Comp., 85, (2016).

[25] M. Prelle and M. Singer, Elementary first integral of differential equations. Trans. Amer. Math. Soc., 279, 215, (1983).

[26] B. van der Pol and J. van der Mark, Frequency demultiplication, Nature, 120, (1927).

[27] G. Duffing, Erzwungene Schwingungen bei Veränderlicher Eigenfrequenz, F. Vieweg u. Sohn, Braunschweig, (1918).

[28] A. Kimiaeifar, A.R. Saidi, G.H. Bagheri, M. Rahimpour and D.G. Domairry, Analytical solution for Van der Pol-Duffing oscillators, Chaos, Solitons and Fractals, 42, (2009).

[29] J. Kyziol and A. Okniński, The Duffing-Van der Pol Equation: Metamorphoses of Resonance Curves, Nonlinear Dynamics and Systems Theory, 15, 1, (2015).

[30] V.K. Chandrasekar, M. Senthilvelan and M. Lakshmanan, New aspects of integrability of forcefree Duffing-van der Pol oscillator and related nonlinear systems, J. Phys. A (Math. Gen.), 37, 16, (2004). 
[31] G. Gao and Z. Feng, First integrals for the Duffing-Van der Pol type oscillator, Eighth Mississippi State - UAB Conference on Differential Equations and Computational Simulations. Electronic Journal of Differential Equations, Conf. 19, (2010), ISSN: 1072-6691. 